\title{
Characteristics of Yearly Fluctuation in the Size of Tile Fish Population in Wakasa Bay and Its Mean Generation Time
}

\author{
Takeru KitaharA* \\ (Accepted July 13, 1984)
}

\begin{abstract}
Analyses were made with yearly fluctuation in tile fish population size in the fishing area of the sweeping trammel net fishery in the central region of Wakasa Bay in order to obtain its characteristics, using the data on the fishery over the period of 1961 to 1981. The estimated population size ranged from 45 to 229 tons and showed a remarkably cyclical pattern with the period of less than five years, 1961-1981. The length and market size category compositions indicated that the cyclical pattern resulted mainly from the great year classes of $1959,1964,1968,1971,1975$ and 1977 and negligibly poor year classes of the other years. Consequently, it follows that the six dominant year classes mainly supported the population, and hence the fishery, in that period. The time intervals between two subsequent dominant year classes are nearly within the mean number of breeding seasons of the female fish for a situation in which fishing pressure was hardly exerted on the population.
\end{abstract}

About 25 years ago, there appeared a new type of fishing gear, sweeping trammel net, called "kogisashi-ami", in Wakasa Bay, Fukui, and it spreaded over other areas such as Ishikawa, Ehime and Shimane. The construction and operation of the gear were described in detail by MAtuda. ${ }^{1)}$ In the sweeping trammel net fishery of Wakasa Bay, over $80 \%$ of total catch is occupied by only tile fish Branchiostegus japonicus japonicus every year and others are Dentex tumifrons and Mustelus manazo etc. ${ }^{2)}$ The fishery has remained relatively flourishing in Fukui and Ishikawa, while in the others, it extincted several years after the respective years when it had begun.

In the sweeping trammel net fishery of Wakasa Bay, annual catches of tile fish have remarkably fluctuated year by year since 1961, the year in which it was begun under a license system, and ranged from 18 to 178 tons over the period of 1961 to 1981 (Table 1). Fig. 1 shows that the peaks of the annual catches appeared in the intervals of less than 5 years in the fishery. Thus, the extinction of the fishery in Ehime and Shimane seems to reflect some characteristics of yearly fluctuation in tile fish population size as stated later. Some authors ${ }^{3-\beta}$ ) tried to discuss such fluctuations in the sizes of exploited fish populations with reference to the characteristics of their life history, for example, age at first maturity, life span, reproductive span and variation of survival rates in early life history etc.
In the present paper, analyses were made with yearly fluctuation in tile fish population size in the fishing area of the sweeping trammel net

Table 1. Annual catches of tile fish in the sweeping trammel net fishery, and the estimated sizes $P$ (in weight) and $N$ (in number) of tile fish population in the fishing area of the fishery in Wakasa Bay from 1961 to 1981

\begin{tabular}{ccrc}
\hline Year & Catch (ton) & \multicolumn{1}{c}{$P$ (ton) } & $N \times 10^{-5}$ \\
\hline 1961 & 89.5 & $156(11)^{\text {* }}$ & 4.6 \\
1962 & 99.5 & $174(12)$ & 5.5 \\
1963 & 88.5 & $154(8)$ & 4.3 \\
1964 & 45.5 & $108(7)$ & 2.7 \\
1965 & 18.1 & $68(7)$ & 1.5 \\
1966 & 29.6 & $61(7)$ & 3.3 \\
1967 & 83.6 & $130(6)$ & 4.5 \\
1968 & 51.0 & $99(7)$ & 2.6 \\
1969 & 29.5 & $45(7)$ & 1.1 \\
1970 & 41.9 & $62(9)$ & 2.4 \\
1971 & 77.4 & $190(15)$ & 6.5 \\
1972 & 37.8 & $84(10)$ & 2.2 \\
1973 & 45.6 & $125(12)$ & 7.4 \\
1974 & 177.8 & $229(5)$ & 7.3 \\
1975 & 82.8 & $149(7)$ & 4.3 \\
1976 & 70.2 & $99(5)$ & 2.2 \\
1977 & 38.7 & $74(6)$ & 2.5 \\
1978 & 63.2 & $123(18)$ & 4.0 \\
1979 & 54.9 & $99(14)$ & 4.8 \\
1980 & 80.7 & $109(9)$ & 3.3 \\
1981 & 47.4 & $66(5)$ & 1.9 \\
\hline
\end{tabular}

* ( ) shows standard error.

* Department of Fisheries, Kyoto University, Kyoto 606, Japan (北原 武: 京都大学路学部水麾学教室). 


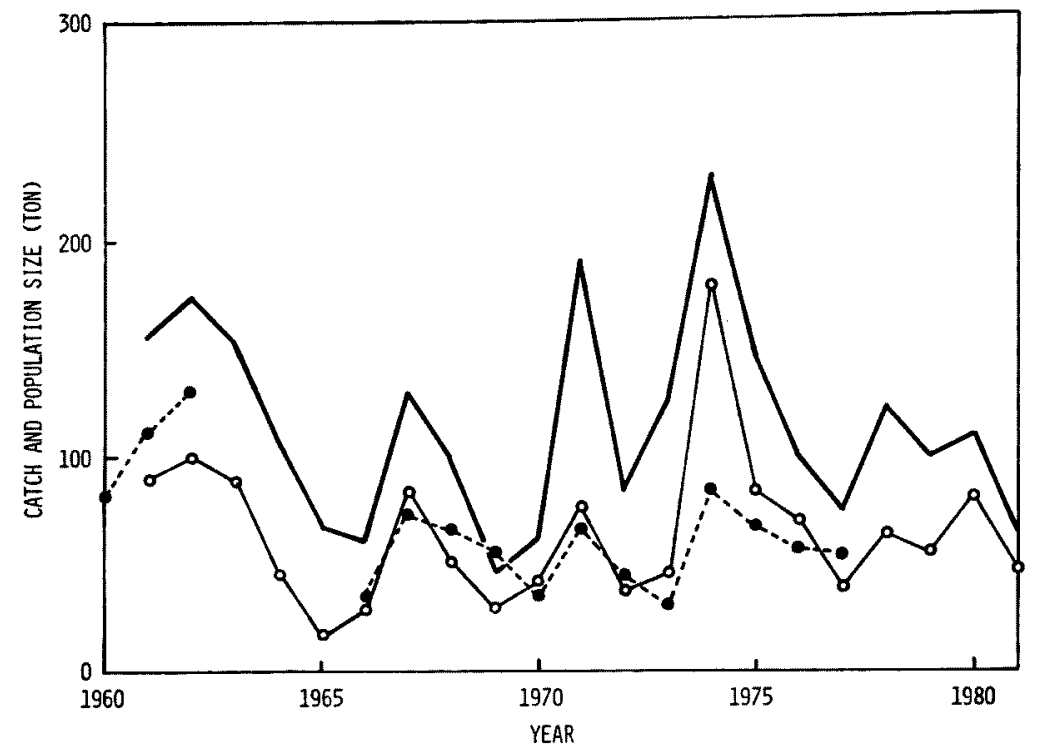

Fig. 1. Annual catches of tile fish by the sweeping trammel net fishery in the central area of Wakasa Bay in Fukui (open circle) and by the long line fishery etc. in the western area of the bay in Kyoto (closed circle), and estimated sizes of tile fish population in the fishing area of the sweeping trammel net fishery from 1961 to 1981 (solid line).

fishery in Wakasa Bay, using the data concerning the fishery over the period of 1961 to 1981 , to obtain its characteristics. Further, this paper attempted to deal with the yearly fluctuation in the population size from a viewpoint of life history characteristics theoretically.

\section{Materials and Methods}

The present paper employed the data on the commercial fishery of tile fish with sweeping trammel net in Wakasa Bay in summer, 19611981. The fishery was licensed in August from 1961 , the year when it was begun under a license system, to 1968 and has been done from Jul. 25 to Sept. 5 since 1969. The fishing area is closed in the central region of Wakasa Bay as shown in Fig. 2 in Matuda's paper ${ }^{7)}$ and its area is about $678 \mathrm{~km}^{2}$. There are regulations for the gear as follows: the length and height of net are less than $300 \mathrm{~m}$ and less than $3.1 \mathrm{~m}$, respectively, and the respective mesh sizes of inner and outer webbings are more than $6 \mathrm{~cm}$ and more than $30 \mathrm{~cm}$ in stretched measure. Fukui Pref. has systematically collected the catch statistics of the fishery, which contain the daily tile fish catches classified by market size categories by every boat, since 1961 . The respective market size categories are large (above $600 \mathrm{~g}$ ), medium (400-600 g), small (200$400 \mathrm{~g}$ ) and extremely small (below $200 \mathrm{~g}$ ) sizes.

Tile fish population sizes were estimated from catch per boat-day during 3 days (CPUE) by modified DELURY's method. ${ }^{8)}$ In the estimation of the population sizes, a few or several CUPE's plotted against cumulative ctach considerably deviated from a linear trend in some years, especially near the end of the fishing season in the years in which the sizes were poor. Accordingly, the population sizes were estimated except the CPUE's deviating remarkably from linear trends. For such deviations were supposed to be due mainly to bad weather or to the fishing outside the licensed area for poor catch near the end of the season in the years when the population sizes were very small.

This paper used the length compositions* of the commercial tile fish catch by the fishery in 1961, 1963-1968 and 1970. The mesh selectivity curve used is the one determined in the previous paper. ${ }^{\theta)}$ The data are utilized on the body length and weight measurements of the samples of the

* The length composition of 1961 was cited from Rept. on the sweeping trammel net fishery by Fukui Pref. Fish. Exp. Stat. (1961) and the one of 1963 was estimated from the catches classified by the categories and the length compositions within categorics. 


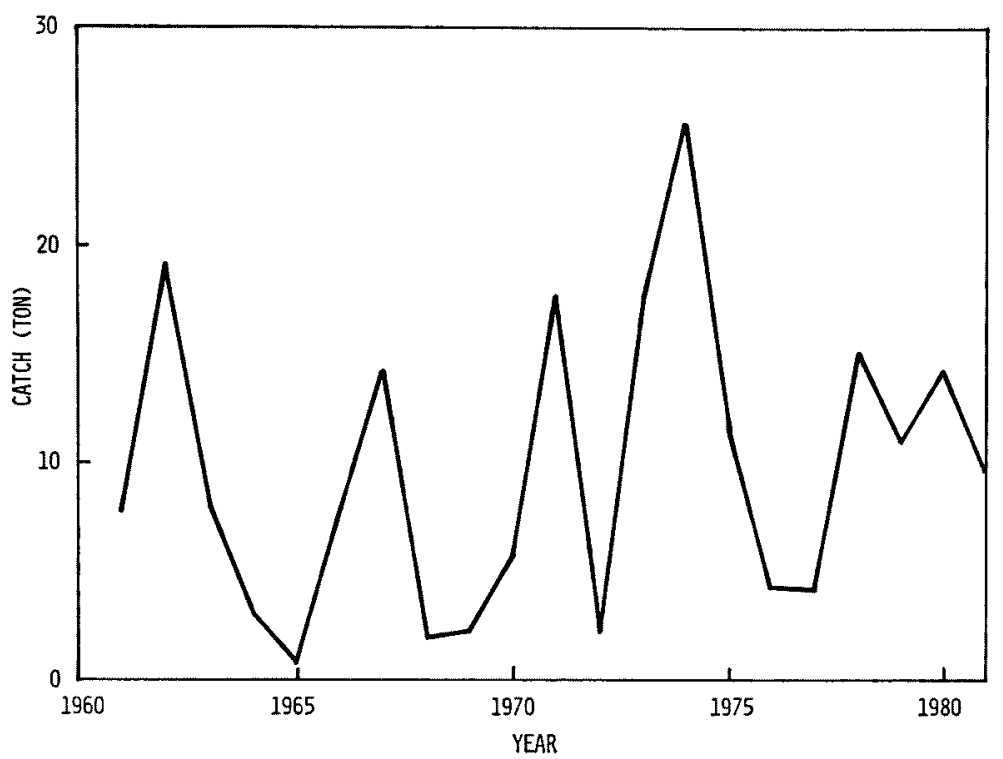

Fig. 2. Annual catches of extremely-small-sized tile fish in the sweeping trammel net fishery in Wakasa Bay from 1961 to 1981.

species taken in 1965.

Tile fish population number $N$ in the area was calculated from the length composition, the catches classified by the size categories and the estimated population size $P$ in weight for each year of 1961 , 1963-1968 and 1970 by the following method. The body length and weight measurements give above $31,27-31,22-27$ and below $22 \mathrm{~cm}$ as the rough length ranges of large, medium, small and extremely small sizes, respectively. Accordingly, it is permitted to assume that the species are classified into the categories according to their lengths. Under the above assumption, first let us treat an index of $n_{j}$ of the number $N_{j}$ of the fish belonging to category $j$ in the population of a given year for brevity. Letting the proportions for length $l_{i}$ in the length composition of the catch and in the one corrected by the mesh selectivity curve by $F_{i}^{\prime}$ and $F_{i}$, the mean body weights $\bar{w}_{j}^{\prime}$ and $\bar{w}_{j}$ of category $j$ in the catch and the population can be given, respectively, by

$$
\bar{w}_{j}^{\prime}=\sum w_{i} F_{i}^{\prime} / \sum F_{i}^{\prime},
$$

and

$$
\bar{w}_{j}=\sum w_{i} F_{i} / \sum F_{i},
$$

where $w_{i}$ refers to body weight of the fish of length $l_{i}$. The mean mesh selectivity $\bar{S}$, for the fish of category $j$ can be presented by

$$
\vec{S}_{j}=\sum S_{i} F_{i} / \sum F_{t},
$$

in which $S_{i}$ is mesh selectivity for the fish of $l_{i}$. Denoting the catch in weight of category $j$ by $C_{j}$, the catch $N_{j}^{\prime}$ in number of $j$ is calculated by the following expression,

$$
N_{j}^{\prime}=C_{j} / \bar{w}_{j}^{\prime},
$$

and hence $n_{j}$ is defined as follows:

$$
n_{j}=N_{j}^{\prime} / \bar{S}_{j} \text {. }
$$

If fishing intensity is assumed to be uniformly exerted on the fish of every category, the mean body weight $\bar{W}$ of the whole population is given by

$$
\bar{W}=\sum \bar{w}_{j} n_{j} / \sum n_{j},
$$

and, consequently, the population number $N$ can be calculated by $N=P / \bar{W} . \quad N_{f}$ is presented by $N_{f}=k n_{j}$, where $k$ is a constant dependent on exploited rate for the population and is determined by a relation, $k \sum \bar{w}_{f} n_{j}=P$.

In the other years in which the length compositions were not obtained, the means of $\bar{w}_{j}{ }^{\prime}, \bar{w}_{j}$ and $\bar{S}_{j}$ for the years stated above were used for the rough estimation of the population numbers by the same method under the assumption that the length compositions little changed within every category among years.

\section{Results}

Table 1 and Fig. 1 show the estimated tile fish 
population sizes in the fishing area of the sweeping trammel net fishery from 1961 to 1981. The population size remarkably fluctuated year by year and it ranged from 45 to 229 tons. The peaks of the population size occurred in irregular intervals below five years, or in 1962, 1967, 1971, 1974, 1978 and 1980. As clear from Fig. 1, the annual catches of the species by the fishery relatively fluctuated year by year in a manner quite similar to the population size. The annual catches of extremely-small-sized fish are plotted against year from 1961 to 1981 in Fig. 2, which indicates that their fluctuation is relatively great as compared with those in Fig. 1 although they are very similar to one another. Taking account of mesh selectivity, such yearly fluctuations may suggest that dominant year classes intermittently occurred.

In Table 1, are given the estimated population numbers of tile fish in the area in 1961, 1963-1968 and 1970 . In the above estimation of the population numbers, it was found that $\bar{w}^{\prime}, \bar{w}$ and $\bar{S}$ kept comparatively constant among years in the three categories except extremely small size in which they were far smaller in 1961, 1966 and 1970 than the other years as shown in Table 2. The years of 1961,1966 and 1970 are the ones before observing the peaks in the annual catches of extremely-smallsized fish in Fig. 2. The findings mean that a dominant year class recruited into exploited phase in each of those years and it was fished mainly in the following year. Consequently,
Fig. 2 also gives the suggestion that a dominant year class presumedly recruited in each of the years before recognizing the peaks in the annual catches of extremely-small-sized fish, or in 1973, 1977 and 1979. For this reason, the population numbers were calculated for 1962, 1969 and 19711981 using, as $\bar{w}^{\prime}, \bar{w}$ and $\bar{S}$ of extremely small size, $\bar{w}^{\prime}=141 \mathrm{~g}, \bar{w}=101 \mathrm{~g}$ and $\bar{S}=0.28$ in 1973 , 1977 and 1979 and $\bar{w}^{\prime}=178 \mathrm{~g}, \bar{w}=179 \mathrm{~g}$ and $\bar{S}=$ 0.75 in the other years. The estimated population numbers are shown in Table 1 and Fig. 3.

Fig. 3 indicates that the population number markedly fluctuated year by year in irregular intervals below five years and ranged from $1.1 \times 10^{5}$ to $7.4 \times 10^{5}$. It should be especially noticed that logarithms of the population number nearly linearly declined in the periods of 1963-1965, 19671969, 1974-1976 and 1979-1981 and that regression lines are nearly parallel to one another in Fig. 3. The finding suggests that the annual recruitments of tile fish were negligibly small in the years when logarithms of the population number linearly declined.

Fig. 4 shows the annual length compositions of the population, which are given by the product of $F_{i}$ and $N$, in 1961, 1963-1968 and 1970. This figure indicates that there appeared the dominant year classes with a mode ' $14-16 \mathrm{~cm}$ length and they were clearly observed at least during two years after every appearance of them. Judging from the growth curve of the species, ${ }^{10), *}$ the re-

Table 2. $\quad \bar{w}^{\prime}, \bar{w}$ and $S$ for each of market size categories in 1961, 1963-1968 and 1970

\begin{tabular}{|c|c|c|c|c|c|c|c|c|}
\hline Year & 1961 & 1963 & 1964 & 1965 & 1966 & 1967 & 1968 & 1970 \\
\hline & \multicolumn{8}{|c|}{ Large Size } \\
\hline $\bar{w}^{\prime}(\mathrm{g})$ & 852 & 718 & 747 & 717 & 684 & 711 & 674 & 687 \\
\hline $\bar{w}(g)$ & 871 & 730 & 761 & 725 & 687 & 723 & 676 & 687 \\
\hline \multirow[t]{2}{*}{$S(\%)$} & 30 & 34 & 33 & 34 & 35 & 33 & 36 & 35 \\
\hline & \multicolumn{8}{|c|}{ Medium Size } \\
\hline $\bar{w}^{\prime}(\mathrm{g})$ & 517 & 457 & 503 & 505 & 505 & 459 & 461 & 485 \\
\hline $\bar{w}(\mathrm{~g})$ & 528 & 458 & 512 & 515 & 516 & 462 & 468 & 497 \\
\hline \multirow[t]{2}{*}{$S(\%)$} & 49 & 61 & 50 & 50 & 50 & 58 & 57 & 53 \\
\hline & \multicolumn{8}{|c|}{ Small Size } \\
\hline $\bar{w}^{\prime}(\mathrm{g})$ & 295 & 296 & 284 & 290 & 291 & 264 & 284 & 263 \\
\hline $\bar{w}(\mathrm{~g})$ & 302 & 303 & 287 & 295 & 295 & 266 & 290 & 267 \\
\hline \multirow[t]{2}{*}{$S(\%)$} & 90 & 90 & 93 & 92 & 92 & 97 & 92 & 96 \\
\hline & \multicolumn{8}{|c|}{ Extremely Small Size } \\
\hline$\overline{\bar{w}^{\prime}}(\mathrm{g})$ & 142 & 182 & 180 & 181 & 129 & 165 & 188 & 152 \\
\hline $\bar{w}(\mathrm{~g})$ & 110 & 166 & 167 & 176 & 97 & 158 & 184 & 97 \\
\hline$S(\%)$ & 31 & 71 & 74 & 80 & 23 & 67 & 84 & 29 \\
\hline
\end{tabular}

* Kryono et al.: Unpublished. 


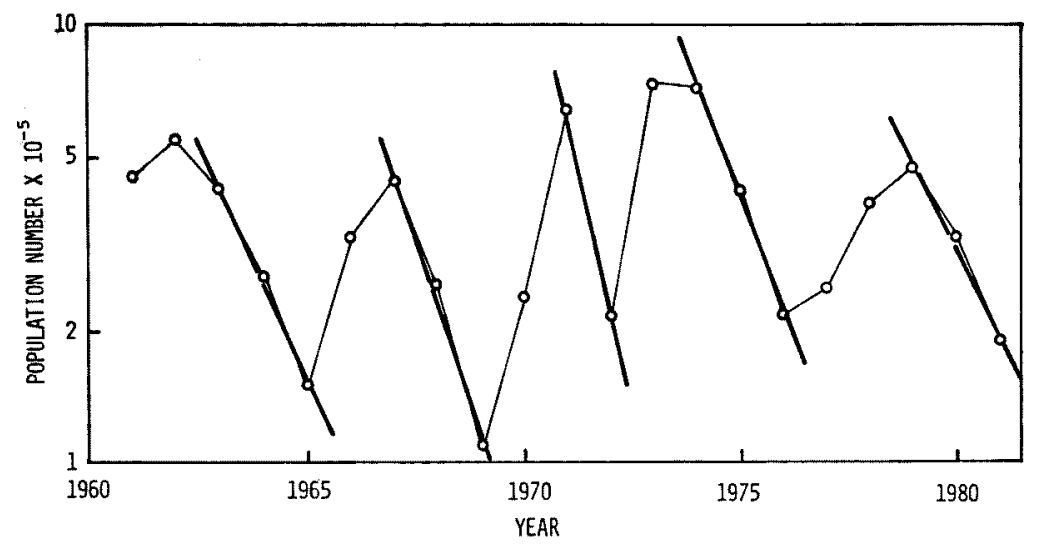

Fig. 3. Yearly fluctuation in the population number of tile fish in the sweeping trammel net fishery in Wakasa Bay from 1961 to 1981 . Thick line shows regression lines.

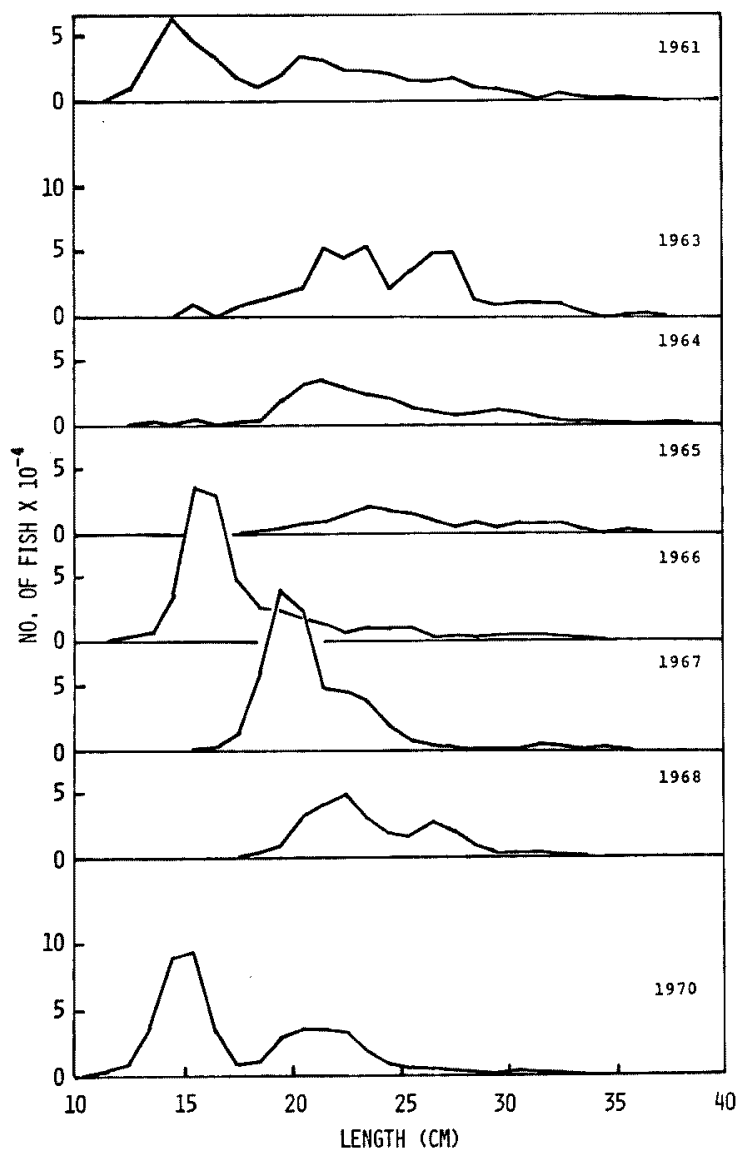

Fig. 4. Annual length compositions of tile fish population in the fishing area of the sweeping trammel net fishery in Wakasa Bay in 1961, 1963-1969 and 1970. 


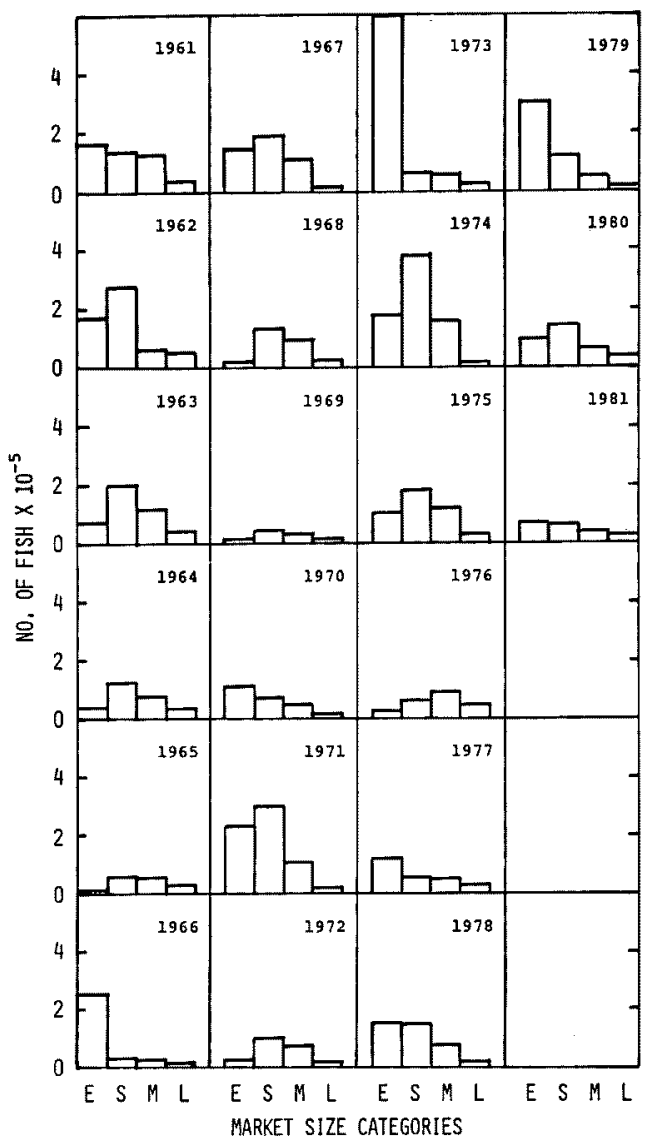

Fig. 5. Annual market size category compositions of tile fish population in the fishing area of the sweeping trammel net fishery in Wakasa Bay over the period of 1961 to 1981 . E, S, M and L show extremely small, small, medium and large sizes, respectively.

spective year classes with a mode of $14-16 \mathrm{~cm}$ length in 1961, 1966 and 1970 are considered to be those of 1959, 1964 and 1968 because age group with mean length of $14-16 \mathrm{~cm}$ in August is $1+$ years old. Fig. 4 also indicates that at least the part of 1 +years old fish recruits into exploited phase. A comparison between Figs. 1 and 4 tells, therefore, that the peaks of the annual catches appear when the ages of the dominant year classes reach $2+$ years old.

The market size category compositions $\left(N_{j}\right)$ are presented over the period of 1961 to 1981 in Fig. 5. Extremely small size consists of the age groups of $1+$ and $2+$ years old and the others contain those of more than two years old. It is clear from Fig. 5 that the several dominant year classes occurred in irregular intervals below five years. That is, generally the number of extremely-smallsized fish gradually declined with year after having reached a peak and steeply increased in a certain year, reaching the next peak in that year or in the following year. The steep increases in the number of extremely-small-sized fish suggest that at least the parts of the dominant year classes recruited into exploited phase. As mentioned previously, therefore, Fig. 5 shows that the dominant year classes recruited in 1973, 1977 and 1979 over the period of 1971 to 1981 , too, and they are those of 1971, 1975 and 1977.

As far as Figs. 4 and 5 are concerned, it is likely that the year classes were presumedly remarkably poor except for the ones of 1959, 1964, 1968, 1971, 1975 and 1977. In the other words, it is the six strong year classes that chiefly supported the population and hence the fishery over the period of 1961 to 1981 . Since fishing intensities exerted on age groups possibly change in accordance with their relative aboundances, ${ }^{11)}$ however, there remains a possibility that a strong age group leads to the overestimation of its aboundance and to the underestimation of others in the method used here.

If it is assumed that the species little either recruited or immigrated in the years when the population numbers in log linearly declined (Fig. 3 ), the regression lines give $0.59,0.49,0.34,0.55$, and 0.63 as estimates of annual survival rates for 1963-1965, 1967-1969, 1971-1972, 1974-1976 and 1979-1981, respectively. Consequently, the upper linit of the annual survival rates may be supposed to be at most $0.5-0.6$ in the population.

\section{Discussion}

The above analyses indicated that the aboundance of tile fish remarkably fluctuated year by year and showed a cyclical pattern with the period of less than five years in the area of the sweeping trammel net fishery in Wakasa Bay from 1961 to 1981. The cyclical pattern resulted mainly from the great year classes of 1959, 1964, 1968, 1971, 1975 and 1977 and negligibly small year classes of the other years. It follows from the above mention that the six dominant year classes almost supported the fishery in the bay over the period of 1961 to 1981. In Ehime and Shimane, therefore, the extinction of the fishery may be considered to be due to its having been unable to endure to a certain year in which the following dominant year 
class recruited. It has been well known that fluctuations in the sizes of exploited fish populations with several age groups are ascribable to the accurrences of dominant year classes and that they give usually good catches (for example, HJORT, ${ }^{12)}$. JONES ${ }^{\text {(1) }) . ~}$

In many studies on demersal fish populations, generally, fluctuations in the sizes of year classes have been treated chiefly from the aspect of stock and recruitment relationship (for example, Beverton and Holt, ${ }^{14}$ ) SaIshu, ${ }^{15)}$ Garrod ${ }^{10}$ ). In the tile fish population, however, the intermittent occurrences of the dominant year classes suggest that the sizes of year classes hardly regulated by stock and recruitment relationship although it is always not denied in a theoretical sense. That is, their intermittent occurrences represent that other factors play great roles in the reproductive process of the species rather than stock and recruitment relationship. The adult females of the species scarcely grow $^{10)}$ and so the reproduction of the female population depends substantially on the small-sized female population. In the years when the dominant year classes were reproduced, the numbers of the small-sized fish ranged from $6 \times 10^{4}$ to $3 \times 10^{5}$.

Before going further, let us pay our attention to theoretical studies on the advantages of iteroparity. ChaRnOV and SCHAFFER ${ }^{17)}$ theoretically predicted that interoparity would be favored by adult survival rates which were high relative to ones of juvenile. On the other hand, MURPHY ${ }^{3)}$ concluded from computer simulations that uncertainty in survival from zygote to first maturity, from whatever source, generated selective pressure for interoparity. SCHAFFER ${ }^{4)}$ examined and proved MURPHY's conclusion with a simple model.

Judging from pronouncedly great mortality rates and/or their great variations in early life history, the hypotheses proposed above seem to be valid for most fish populations. In the tile fish population, the time intervals between two subsequent dominant-year-classes have to be related with its mean generation time if their occurrences result from yearly variation of survival rates in the early life history according to MurPHY and SCHAFfer. Accordingly, the mean generation time $\bar{T}$ of the female population is estimated in the following.

Mean generation time $\bar{T}$ is defined as follow:

$$
\bar{T}=\sum_{i=m}^{d} i(1-D)^{i-m} g_{i} / \sum_{i=m}^{d}(1-D)^{i-m} g_{i}
$$

in which $m$ is age at first maturity, $d$ maximum age, $D$ annual adult mortality rate (constant) and $g_{i}$ gonad weight at age $i$ in the female population. Eq. (7) gives 6.4, 5.7, 5.0 and 4.4 years as the mean generation times for $D$ of $0.2,0.3,0.4$ and 0.5 , respectively. Now, the mean number $\bar{T}^{*}$ of breeding seasons, or the years during which an adult female contributes to reproduction, is defined as $T^{*}=\bar{T}-m+1$. Because most females of more than 2 years old take part in reproduction, ${ }^{18)} \bar{T}^{*}$ is estimated to be 5.4 and 4.7 years for natural mortality rates of 0.2 and 0.3 that are rough obtained with TANAKA's formula. ${ }^{19)}$ Thus, the time intervals between two subsequent dominant year classes are nearly within the estimates of $\bar{T}^{*}$ for the period when fishing pressure was hardly exerted on the population. The above result means that an adult female, on an average, has at least a good year for reproduction between the ages at first maturity and mean generation time in the absence of intense fishings in varying environments. In such a sense, $\bar{T}^{*}$ is possibly available for diagnosing exploited fish populations consisting mainly of strong year classes that intermittently occur.

An increase in fishing or predation pressure naturally reduces the mean generation time of a target fish population and, under the above hypothesis, may somewhat change its life history characteristics in such cases that it little has influence on the population size, etc. For instance, if age at first maturity is more or less different among the members of a population and if their relative advantages keep a balance among them in irregularly varying conditions, there is a possibility that rather earlier reproduction is favored by an increase in fishing or predation pressure. In practice, the length at first maturity markedly declined in the Alaska pollack Theragra chalcogramma population, whose annual catches steeply increased from the middle 1960's, in the eastern region of Bering Sea in the early 1970 's. ${ }^{20}$ Power et al. ${ }^{21)}$ reported that, in lake trout Salvelinus namaycush in the lake where a seal population inhabited, the old fish were unable to be observed, ages at first maturity low and spawned eggs small but many as compared with ones in lakes without it.

As seen from Fig. 1, because the catches of tile fish in Kyoto Pref, relatively show a nearly similar tendency to the ones by the sweeping trammel net fishery in the central region of Wakasa Bay, the estimated population sizes of the species in the area may be used as an index of the population sizes all over the bay.

Finally, the author wishes to express his sincere 
thank to Dr. H. KAWAI for his supporting this work. The author is also deeply grateful to Mr. K. OE, Fish. Division of Fukui Pref., for placing the data concerning the catch statistics of the tile fish fishery.

\section{References}

1) K. Matuda: Mem. Coll. Agr. Kyoto Univ., 103, 1-17 (1972).

2) T. Krtahara and K. Matuda: Bull. Japan. Soc. Sci. Fish., 33, 1087-1091 (1967).

3) G. I. Murphy: Amer. Natur., 102, 391-403 (1968).

4) W. M. SChaffer: Amer. Natur., 108, 318-323 (1974).

5) D. A. Roff: Can. J. Fish. Aquat. Sci., 38, 968977 (1981).

6) T. KawaSakI: Bull. Japan. Soc. Sci. Fish., 48, 605-609 (1982).

7) K. Matuda: Bull. Japan. Soc. Sci. Fish., 33, 1092-1095 (1967).

8) T. Krtahara: Bull. Japan. Soc. Sci. Fish., 48, 1389-1395 (1982).

9) T. Kutahara: Bull. Japan. Soc. Sci. Fish., 34, 759-763 (1968).
10) Y. Hayashi: Bull. Japan. Soc. Sci. Fish., 42 , 1243-1249 (1976).

11) T. Krtahara: Bull. Japan. Soc. Sci. Fish., 39, 471-476 (1973).

12) J. HJort: J. Cons. Int. Explor. Mer, 1, 6-38 (1926).

13) R. Jones: J. Cons. Int. Explor. Mer, 41, 50-62 (1983).

14) R. J. Beverton and S. J. Holt: On the dynamics of exploited fish populations, Fish. Inv. Ser. II, U. K., 1957, pp. 44-67.

15) K. SAishu: Jap. J. Ecol., 20, 31-39 (1970).

16) D. J. Garrod: J. Cons. Int. Explor. Mer, 41, 63-66 (1983).

17) E. L. Charnov and W. M. SCHAFFer: Amer. Natur., 107, 791-793 (1973).

18) Y. Hayashi: Bull. Japan. Soc. Sci. Fish., 43, 1273-1277 (1977).

19) S. Tanaka：Bull. Tokai Reg. Fish. Res. Lab., 28, 1-200 (1960).

20) Y. KrTANO: in "Shigen-seibutsu-gaku" (ed. by M. NishiwaKI), Todai-shuppan, Tokyo, 1974, pp. 119-126.

21) G. Power and J. Gregorre: J. Fish. Res. Bd. Can., 35, 844-850 (1978). 